\title{
RadNet 1.0: exploring deep learning architectures for longwave radiative transfer
}

\author{
Ying Liu, Rodrigo Caballero, and Joy Merwin Monteiro \\ Department of Meteorology, Stockholm University, Stockholm, Sweden
}

Correspondence: Ying Liu (ying.liu.sweden@gmail.com)

Received: 25 November 2019 - Discussion started: 21 January 2020

Revised: 11 July 2020 - Accepted: 21 July 2020 - Published: 21 September 2020

\begin{abstract}
Simulating global and regional climate at high resolution is essential to study the effects of climate change and capture extreme events affecting human populations. To achieve this goal, the scalability of climate models and efficiency of individual model components are both important. Radiative transfer is among the most computationally expensive components in a typical climate model. Here we attempt to model this component using a neural network. We aim to study the feasibility of replacing an explicit, physics-based computation of longwave radiative transfer by a neural network emulator and assessing the resultant performance gains. We compare multiple neural-network architectures, including a convolutional neural network, and our results suggest that the performance loss from the use of conventional convolutional networks is not offset by gains in accuracy. We train the networks with and without noise added to the input profiles and find that adding noise improves the ability of the networks to generalise beyond the training set. Prediction of radiative heating rates using our neural network models achieve up to $370 \times$ speedup on a GTX $1080 \mathrm{GPU}$ setup and $11 \times$ speedup on a Xeon CPU setup compared to the a state-of-the-art radiative transfer library running on the same Xeon CPU. Furthermore, our neural network models yield less than $0.1 \mathrm{~K} \mathrm{~d}^{-1}$ mean squared error across all pressure levels. Upon introducing this component into a singlecolumn model, we find that the time evolution of the temperature and humidity profiles is physically reasonable, though the model is conservative in its prediction of heating rates in regions where the optical depth changes quickly. Differences exist in the equilibrium climate simulated when using the neural network, which are attributed to small systematic errors that accumulate over time. Thus, we find that the ac-
\end{abstract}

curacy of the neural network in the "offline" mode does not reflect its performance when coupled with other components.

\section{Introduction}

Computational models of Earth's climate are essential tools to advance our understanding of the climate system and our ability to predict its response to perturbations such as increased levels of greenhouse gases. Climate models contain algorithmic representations of the various components of the climate system like the atmosphere, ocean, sea ice, and land surface. Our ability to predict future changes in climate depends crucially on the accuracy of these models and the extent to which interactions between various components of the climate system are represented.

A basic requirement for increased model fidelity, particularly at the regional scale, is increased spatial resolution. However, the computational burden increases roughly as the fourth power of spatial resolution (since resolution must increase along all three spatial dimensions and the time step reduced to ensure numerical stability). To address this problem, various approaches have been used including improved model scalability (Dennis and Loft, 2011) and the use of lowprecision floating point operations (Palmer, 2014).

Long simulations using high-resolution climate models are needed to explore key questions in climate research, particularly changes in the statistics of weather extremes such as windstorms and precipitation events. Radiative transfer (RT) in the atmosphere is among the most computationally burdensome components of such simulations. While the basic equations for calculating RT are straightforward, the complex nature of the absorption bands of greenhouse gases such 
as carbon dioxide and water vapour requires separate calculation over a very large number of small spectral intervals to obtain accurate results. Since such a line-by-line calculation is extremely computationally intensive and not feasible in a realistic climate model integration, it is necessary to group individual absorption lines into bands or clusters with similar properties as in the correlated-k method (Fu and Liou, 1992). Such methods can dramatically improve the computational performance while retaining adequate accuracy in the computation. Many state-of-the-art climate models use the Rapid Radiative Transfer Model for General circulation models (RRTMG). RRTMG is based on the single-column correlated k-distribution reference model RRTM (Iacono et al., 2008b). RRTMG tries to strike a balance between computational complexity and accuracy by reducing the number of calculations per band while ensuring fidelity with the RRTM code (Iacono et al., 2008a). Nonetheless, even when employing such simplified schemes, RT remains amongst the most numerically expensive components of climate models, and a variety of strategies have been developed to reduce this cost (see for example Pincus and Stevens, 2013, and references therein).

In this paper, we explore the potential performance gains achievable by using a neural network (NN) to calculate radiative transfer. Specifically, we train a variety of alternative $\mathrm{NN}$ architectures on a set of radiative heating rate profiles computed using a state-of-the-art RT code (see Sect. 2) and compare the computational performance of the $\mathrm{NN}$ with that of the RT code itself. Note that this comparison only serves to assess the performance of RT calculation in stand-alone form. We expect a suitably trained neural network to be a drop-in replacement for the RT code in a full climate model and expect that other computational costs - such as data transfer within and between computational nodes - will not change, but we do not explicitly address this issue in this exploratory study. Instead, our focus here is on identifying the most suitable $\mathrm{NN}$ architecture in terms of accuracy and computational performance. We also explore the behaviour of the $\mathrm{NN}$ in a time-evolving, single-column radiative-convective model (Sect. 4).

Recent advances in NNs have led to rapid progress in the accuracy of pattern and image recognition tasks. In particular, convolutional neural networks (CNNs) (Krizhevsky et al., 2012a) have achieved impressive results for image classification (Krizhevsky et al., 2012b), while recurrent neural networks (RNNs) have made breakthroughs in sequenceto-sequence learning tasks such as machine translation (Wu et al., 2016). Efforts to use machine learning techniques to model actual physical processes in a climate model have increased recently (Schneider et al., 2017; Gentine et al., 2018; Rasp et al., 2018; O'Gorman and Dwyer, 2018; Scher, 2018; Brenowitz and Bretherton, 2018, 2019; San and Maulik, 2018; Yuval and O'Gorman, 2020). In particular, it is now being recognised that physical processes whose representation in climate models has usually been inexact and pa- rameterised could potentially be improved by using machine learning techniques. RT, on the other hand, has always been an attractive candidate to optimise in climate models because of the large computational cost, as discussed above. Optimisation has been attempted using traditional optimisation, porting to new architectures such as GPUs (Price et al., 2014; Mielikainen et al., 2016; Malik et al., 2017), and using NNs to approximate RT. Initial attempts to retrieve radiative heating profiles used shallow (one hidden layer) networks (Chevallier et al., 1998), and similar NN architectures were successfully used to replace RT in decadal simulations using conventional climate models (Krasnopolsky et al., 2005, 2008, 2009). Recently, a deep NN was used to replace RT in a high-resolution general circulation model (GCM) and was successfully used to run the GCM for 1 year (Pal et al., 2019). These studies show the capability of NNs to accurately approximate radiative heating profiles in a particular climate regime, while raising questions about how generalisable this learning actually is in terms of handling perturbed climate states. Studying the effect of perturbations (in sea-surface temperature, greenhouse gases, aerosols, or cloud properties) on the climate of a model is a very typical use case in climate science, and the performance of NNs in such scenarios has yet to be studied carefully.

In the context of machine learning for climate modelling applications, the following questions are still not well understood (Dueben and Bauer, 2018):

- What NN architectures are most suitable?

- What is the accuracy-efficiency tradeoff between different $\mathrm{NN}$ architectures?

- What accuracy loss can we expect when the NN is provided with "non-typical" input values, i.e. values very different from those in the training sample, such as would occur in a perturbed climate experiment?

- What is the speedup we can expect by replacing a traditional RT scheme with a NN?

Our aim here is to address these four questions. To limit the scope of this exploratory study, we focus on longwave radiative transfer under clear-sky conditions (henceforth, RT thus refers to clear-sky longwave radiative transfer). We use the RRTMG library available within the climt modelling toolkit (Monteiro et al., 2018) to generate radiative cooling profiles to train the NN models. In particular, we compare the accuracy-computational complexity tradeoff between five kinds of NN architectures on both CPU and GPU. We also study the loss in accuracy if perturbations are added to the input. The question of accuracy loss is all the more relevant in RT due to its mathematical structure - since RT is modelled as an integral equation, localised perturbations have global impacts on the profile of radiative heating or cooling obtained. 
The paper is organised as follows. The preparation of data for training and validation of the NNs is presented in Sect. 2 . Section 3 presents the NN structures and parameters we have used. Evaluation results are presented in Sect. 4. Finally, we present a brief discussion along with concluding remarks in Sect. 5.

\section{Data and methods}

While radiative transfer is inherently three dimensional, increasing its complexity and computationally cost, it is common to assume horizontal homogeneity (independent column assumption) and retain only a single (vertical) dimension (Meador and Weaver, 1980). This independent column assumption underlies almost all radiative transfer codes used in weather and climate models and reduces radiative transfer calculation to an "embarrassingly parallel" one-dimensional problem in each vertical column of the atmosphere. For a given longitude-latitude point, RT can be represented by a vector whose length is the number of vertical levels into which the column is discretised. The calculation of RT under clear-sky (cloud-free) conditions is based on a number of inputs, including vectors of atmospheric pressure, air temperature, and specific humidity at each level, while surface temperature and carbon dioxide mixing ratio are represented as scalars. While the clear-sky RT in the atmosphere is affected by other greenhouse gases like methane and aerosols like sulfates, we restrict ourselves to using the above quantities in this exploratory study.

\subsection{The ERA-Interim dataset}

We use the ERA-Interim dataset (Dee et al., 2011) to provide temperature and humidity profiles for training the neural network. The horizontal resolution of the data is $0.75^{\circ} \times 0.75^{\circ}$ in the horizontal. We use 6-hourly model-level data, which has a higher resolution in the vertical as compared with the pressure level data. The vertical grid is a nonuniform $\eta$-coordinate grid with 60 mid-levels from the surface to $0.2 \mathrm{hPa}$ and 61 interface levels from the surface up to $0.1 \mathrm{hPa}$. This implies that pressure is not a constant and is therefore an additional input to the neural network.

The ERA-Interim dataset consists of 38 years of data spanning the period 1979 to 2016, which amounts to around 6.5 billion sample profiles. We employ the first 7 years of ERA-Interim historical data as the training dataset, i.e. data from 1979 to 1985, and the last 2 years of the ERA-Interim historical data as the validation dataset, i.e. data from 2015 to 2016. Considering the model training time, we have applied random sampling of $1 \%$ with respect to each year in the training and validation datasets. This gives around 12 million training samples and 3.5 million validation samples. After sampling, we name the training dataset as Dataset ${ }_{1}$ and the validation dataset as Dataset ${ }_{1 . v a l}$. The reason for using
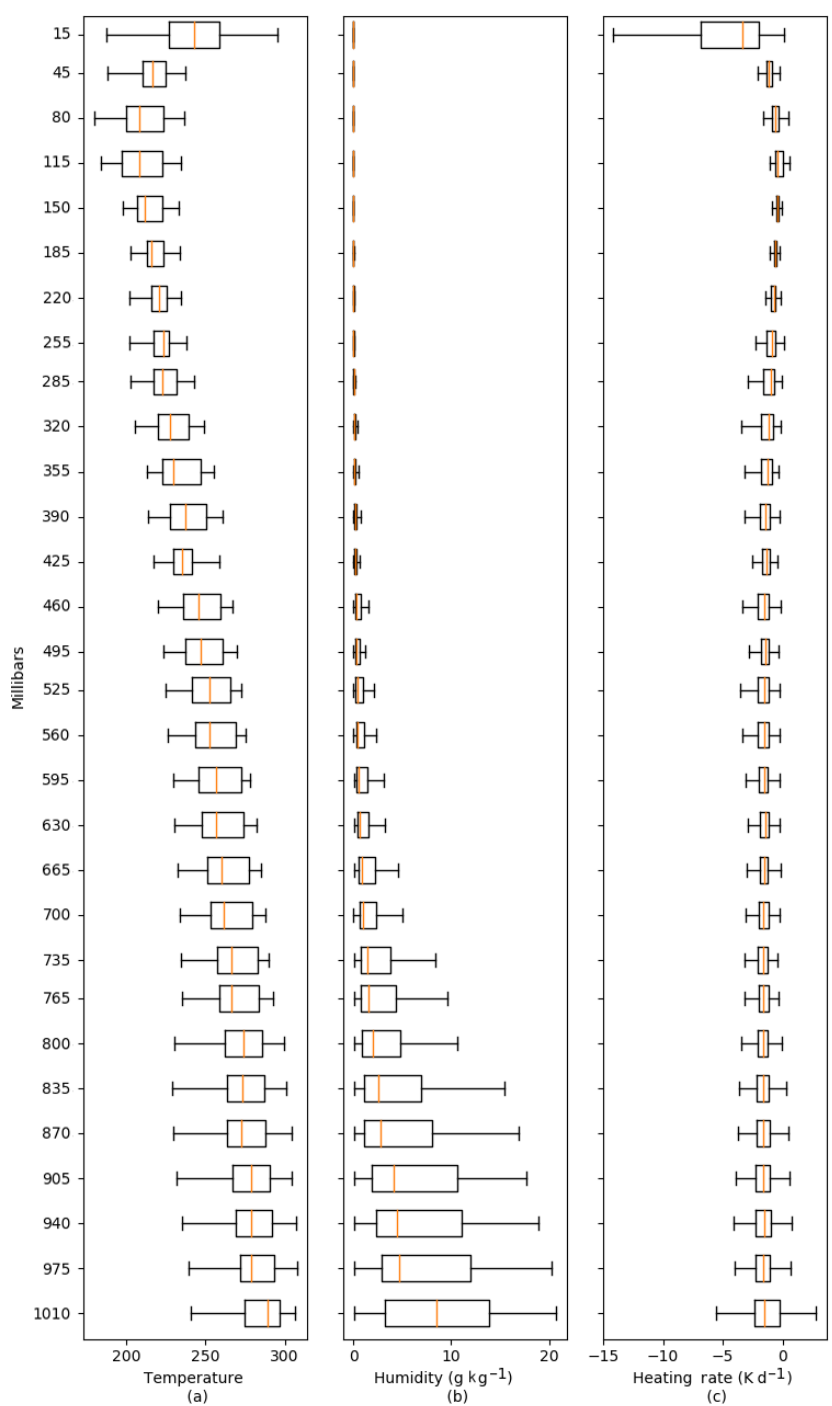

Figure 1. (a, b) ERA-Interim air temperature $(\mathrm{K})$ and humidity $\left(\mathrm{g} \mathrm{kg}^{-1}\right)$ statistics. (c) Longwave radiative rates $\left(\mathrm{K} \mathrm{d}^{-1}\right)$ calculated using RRTMG. Vertical axis is pressure in pascals $(\mathrm{Pa})$.

this data separation schema is because that we would like to examine whether our radiation prediction model is able to generalise to unseen/future data inputs while being trained on the oldest data.

\subsection{Perturbed dataset}

Figure 1 shows the mean and variance in ERA-Interim air temperature, humidity, and radiative heating rates calculated using RRTMG from 1979 to 2016. Using the above statistics, we have augmented our training data by created a perturbed dataset as follows:

1. Pick an original profile from the historical samples. 
2. Generate a random air temperature profile assuming Gaussian distribution at each vertical level using the statistics from Fig. 1.

3. Generate a random weight (between -0.2 and 0.2 ) for the generated air temperature profile.

4. Generate an augmented air temperature profile by adding together the original profile with the weighted random profile vertical-level-wise.

5. Calculate the maximum humidity given the air temperature and pressure at each vertical level.

6. Calculate the original relative humidity ratio using humidity divided by the maximum humidity at each vertical level.

7. Calculate the new maximum humidity given the generated air temperature and pressure at each vertical level.

8. Generate the corresponding humidity by multiplying the new maximum humidity and the original relative humidity ratio at each vertical level.

9. We keep the surface temperature and the carbon dioxide mixing ratio the same as the original profile.

The motivation for adding random, vertically uncorrelated perturbations is that the optical properties of the atmosphere (which determine the radiative heating profiles) can be quite noisy in the vertical. This noisiness is due to the presence of clouds, hydrometeors, aerosols, and horizontal advection of water vapour at different levels in the atmosphere. Changes in optical depth due to the above factors need not have a strong vertical correlation either. The kind of perturbations we have added represent an extreme case of this physically motivated reasoning.

Augmented datasets are generated using Dataset ${ }_{1}$ and Dataset $_{1 . v a l}$. Then, the augmented datasets are 50-50 mixed with Dataset ${ }_{1}$ and Dataset ${ }_{1 . v a l}$ respectively to create Dataset $_{2}$ and Dataset 2.val. The purpose of generating Dataset 2 and Dataset $_{2 . v a l}$ is that we would like to use it to investigate the generality of our RT prediction model. The specific evaluation procedures are described in the evaluation section.

\subsection{The RT dataset}

The calculation of the radiative fields for the historical and perturbed datasets are calculated using the RRTMG component available in the climt modelling toolkit (Monteiro and Caballero, 2016; Monteiro et al., 2018). This component is a Python wrapper over the RRTMG Fortran library and provides convenient access to the radiation fields. The statistics of the generated radiative heating profiles are also shown in Fig. 1.

\section{Neural network models}

\subsection{Neural network basics}

A neural network is composed of multiple neurons - or even multiple layers of neurons - in order to model complex scenarios. A simple neural network is a feedforward network where information flows only in one direction from input to output. Multilayer perceptron (Gardner and Dorling, 1998) is the most common feedforward NN. It consists of an input layer that passes the input vector to the network, one or more hidden layers, and an output layer. There are usually activation functions applied in each layer. An activation function usually introduces nonlinearity in order to allow a $\mathrm{NN}$ to tackle with complicated problems and learn complex representations.

Convolutional $\mathrm{NN}$ is another type of $\mathrm{NN}$ designed for image-focused tasks. It is widely used in many fields such as image classification, object detection, and image segmentation (Krizhevsky et al., 2017). CNNs usually consist of three types of layers, convolutional layers, pooling layers, and fully connected layers. A convolutional layer is composed of learnable kernels or filters. The kernel usually considers a small region of input at one time but covers the entirety of the input. Specifically, it slides over the input spatially and computes dot products between the kernel and the area of input covered by the kernel. With each kernel, a convolutional layer produces an activation map, whose size depends on whether there is a stride or padding. All the activation maps will be stacked together along the depth dimension and passed on to the next layer (O'Shea and Nash, 2015). Neurons in a layer are connected to only a small region of the previous layer instead of everything, which is different from feedforward neural networks. In this way, convolutional layers are better at extracting locality-dependent features, such as shapes and patterns in images.

In the context of RT, we use CNNs to evaluate whether the sensitivity to localised features improves the prediction performance of deep neural networks. In particular, strong local changes in the optical properties of the atmosphere are fairly common due to the presence of clouds or horizontal advection of water vapour. While this work focuses on clearsky radiation, we study the ability of CNNs to recognise and respond to such local features in the single-column simulations.

Table 1 illustrates the structures and parameters of our neural networks. Specifically, we have designed five neural networks, including two feedforward neural networks and three convolutional neural networks (CNN). The input data to the CNNs are prepared by concatenating different variables along the second dimension instead of using different variables as "channels". Therefore, the CNNs use twodimensional convolutional filters instead of one-dimensional filters. Model A and Model B are implementations of feedforward neural networks with different numbers of layers and 
of neurons in each layer. Model C is a simplified CNN implementation based on previous work (Simonyan and Zisserman, 2014). The stride of convolutional filters is set to 1 so that the convolutional filters go through the input array with one element each step. We have not applied any padding to the input. We have not used pooling layers in between convolutional layers, and the convolutional filters are the classic $3 \times 3$ filters. Model D is a variant of Model C with one more convolutional layer. Model $\mathrm{E}$ has the same neural network structure as Model C. The only difference is that Model E has padded the input with an edge of zeros to emphasise on edges of the input. We have used TensorFlow 1.8.0 library for the neural network implementation.

In addition to the above models, we also evaluate a variant of Model E denoted as Model F. Model F is based on Model $\mathrm{E}$ but with a fixed pressure grid. This means that Model F does not take pressure values as input and interpolates air temperature and humidity from model levels onto a fixed, time-invariant pressure grid. While this configuration reduces the dimensionality of the input, it requires extrapolation of the ERA-Interim data or the calculated/predicted RT to the fixed grid. Specifically, the inputs of a sample profile are B-spline extrapolated according to a fixed pressure grid. We extrapolate the air temperature and humidity values onto the fixed pressure grid based on the profile's pressure range. The inputs corresponding to the rest of the pressure levels are set to 0 . After running through Model F, the RTs on the static pressure grid are B-spline interpolated back to the original pressure levels, which are the final results. It is important to mention that we constructed the static grid using 15 equally spaced pressure levels from 1 to $500 \mathrm{~Pa}$, another 15 equally spaced pressure levels from 550 to $50000 \mathrm{~Pa}$, and 30 equally spaced pressure levels from 50300 to $103000 \mathrm{~Pa}$. We made this design choice by observing the distribution of the ERAInterim data to ensure that our fixed grid encompasses most common pressure profiles in order to achieve a better accuracy on the extrapolation and interpolation. We used Model F to run the single-column model simulation presented below, which employs a fixed pressure grid.

\subsection{Model training}

We trained our five NNs with two datasets, resulting 10 different models. The first dataset is the aforementioned ERAInterim dataset, namely, Dataset ${ }_{1}$. The second dataset is the augmented dataset, i.e. Dataset ${ }_{2}$, in order to generalise the model to a wider operational region beyond Dataset ${ }_{1}$. Each neural network is trained using the training dataset of either Dataset $_{1}$ or Dataset $_{2}$ and validated against either Dataset ${ }_{1 . v a l}$ or Dataset 2.val.

Each model was trained with 30 epochs under a batch size of 128 starting with a learning rate of 0.001 , which then exponentially decays every 10 epochs with base 0.96 . This setup was empirically obtained, while we observe that all models have converged after the training. Mean squared error is used as the loss function in all models. Parametric rectified linear units (PReLUs) (He et al., 2015a) are used as activation functions in all models since PReLUs is able to resolve the problem of vanishing gradient during model training. The Adam optimiser (Kingma and Ba, 2014) is employed to compute the gradients.

We present the evaluation results regarding the performance of these models in the next section.

\section{Evaluation}

\subsection{Evaluation setup}

We prepared two datasets, i.e. Dataset ${ }_{1 . v a l}$ and Dataset ${ }_{2 . v a l}$, to evaluate our neural network models. Dataset $t_{1 . v a l}$ is used to evaluate the accuracy of the trained models with realistic future data. Dataset 2 .val is used to evaluate the generality of the trained models as it contains profiles that are perturbed versions of the ERA-Interim data.

\subsection{Prediction accuracy}

We use vertical level-wise root-mean-squared errors (RMSE) to compare our $\mathrm{NN}$-generated radiative cooling rates with those generated by the RRTMG algorithm. Figures 2-5 present results for the different NN models. The RMSE is calculated by taking the difference between $\mathrm{NN}$ - and RRTMG-calculated radiative cooling profiles.

Figure 2 presents the RMSE when the NN models are trained using Dataset ${ }_{1}$ and validated against Dataset $_{1 . v a l}$. These experiments are performed to evaluate the capability of different NN models to predict RT when the atmospheric profiles are sampled from the ERA-Interim dataset itself. We see that a simple three-layer feedforward neural network (Model A) is able to predict heating rates with a median RMSE of less than $0.01 \mathrm{~K} \mathrm{~d}^{-1}$ across all pressure ranges. The performance does not improve when more layers of directly connected neurons are added, as shown by Model B. We observe significant RMSE improvement while using CNNs (Models C, D, E). However, the performance differences among these three CNN models are not substantial except the RMSEs near the surface, which tend to have higher variability as shown in the statistics in Fig. 1. Since surface radiation is particularly important to the climate, efforts have been made to minimise its prediction error. The input matrix of Model $\mathrm{C}$ is padded with zeros in order to allow convolutional filters to put equal emphasis on the edges values as the middle ones (Innamorati et al., 2018). This creates Model E, which shows much better prediction accuracy on the bottom and top pressure levels.

Figure 3 presents the RMSE when the NN models are trained using Dataset ${ }_{2}$ and validated against Dataset $_{1 . v a l}$. In this experiment, we examine whether it is possible to expand the operational region of the NN models without compromising on their performance on the ERA-Interim dataset. Com- 
Table 1. Neural network models used for predicting RTs. "fc-X" represents a fully connected layer with X number of neurons."1conv-YxY$\mathrm{X}$ ” represents a convolutional layer with X number of YxY filters.

\begin{tabular}{llllllll|r}
\hline $\begin{array}{l}\text { Model } \\
\text { name }\end{array}$ & \multicolumn{9}{c}{ Model structure } & & & $\begin{array}{r}\text { Number of } \\
\text { parameters }\end{array}$ \\
\hline Model A & input-60x4 & fc-512 & fc-1024 & fc-512 & output-60 & & & 1202176 \\
Model B & input-60x4 & fc-512 & fc-1024 & fc-2048 & fc-1024 & fc-512 & output-60 & 5396480 \\
Model C & input-60x4 & conv-3x3-128 & conv-3x3-256 & fc-512 & output-60 & & & 7666816 \\
Model D & input-60x4 & conv-3x3-128 & conv-3x3-256 & conv-3x3-256 & fc-512 & output-60 & & 7994496 \\
Model E & input-62x6 & conv-3x3-128 & conv-3x3-256 & fc-512 & output-60 & & & 15531136 \\
Model F & input-62x5 & conv-3x3-128 & conv-3x3-256 & fc-512 & output-60 & & & 7928960 \\
\hline
\end{tabular}

paring to Fig. 2, we see that the increased generality comes at the cost of roughly doubled RMSE across all models.

The improvement on generality is suggested by the results shown in Figs. 4 and 5 when the NN models are trained using Dataset $_{1}$ or Dataset 2 and validated against Dataset $_{2 . v a l}$. The RMSE increases by almost 100 times across all models trained with Dataset ${ }_{1}$ and validated against Dataset $_{2 . v a l}$ (Fig. 4) when compared to their validation against Dataset 1.val (Fig. 2). This suggests that models trained with Dataset ${ }_{1}$ cannot really generalise to predict heating profiles from Dataset ${ }_{2 . v a l}$. On the other hand, the RMSE increases 10 times when the models are trained using a wider range of data, i.e. Dataset 2 , as shown in Fig. 5. This is mainly because that the model needs to cover a larger operational region.

When trained on Dataset 1 and validated against

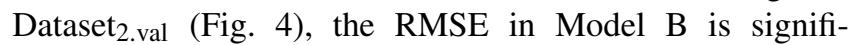
cantly higher. This observation leads us to believe that Model B is more likely to overfit the training dataset. Given that Dataset $t_{2}$ is more perturbed than Dataset $_{1}$ and more parameters and layers in Model B, the nature of feedforward NN (Goodfellow et al., 2016) makes Model B more deeply coupled with patterns observed in the training data ( Dataset $_{1}$ ), which leads to larger errors while evaluating against Dataset $t_{\text {2.val }}$.

Model F displays significant errors on both edges of the pressure levels. This is due to extrapolation errors. Specifically, if the lowest pressure level in an atmospheric profile is lower than the lowest pressure level of the fixed grid, the profiles need to be extrapolated. The same issue arises with the highest pressure levels as well. Thus, the errors in Model F are mainly due to these extrapolation-based artefacts rather than an issue with the training itself. In fact, this model provides the most stable time integration of the single-column model.

In the above evaluations, we have shown that CNN-based models achieve much lower prediction RMSE than feedforward NN models. However, in the next section, we show that $\mathrm{CNN}$-based models tend to have much slower prediction speed, i.e. less speedup as compared with the feedforward models.

\subsection{Prediction speed}

In this section we compare the computation time of RRTMG and NN models using GPUs and CPUs. These performance evaluations have been performed using Intel Xeon CPU E31230 v5 @ 3.40 GHz, Nvidia GTX 1060 GPU with 6 GB of onboard memory, and Nvidia GTX 1080 GPU with 8 GB of onboard memory. Both GPUs we use are commodity hardware and are easily available in the market. RRTMG was run in a single-threaded mode for the purposes of this evaluation.

Table 2 summarises the speedups using NN models to predict RT as compared to RRTMG. The calculation time of NN code and RRTMG code is profiled using the Python line_profiler based on cProfile. The execution time results are averaged from 10 measurements with execution of 100000 predictions per measurement. Since RT calculations are embarrassingly parallel, we are able to use batch predictions in our NN models while using a single GPU. The overall results show that the larger the batch size, the larger the speedup observed as long as the CPU or GPU memory is sufficient. In other words, the calculation of $M$ radiative heating profiles is faster than $M$ times the time taken to predict one such profile. This is because of the efficiency of matrix multiplications in NNs while conducting NN forward pass in batches. We note that such a speedup is not possible in a physicsbased RT scheme since the calculation of RT for an arbitrary atmospheric profile cannot be expressed as a simple matrix multiplication.

The results show that by using only the Xeon CPU, NN Models A and B are able to achieve speedups up to $10.88 \times$ and $2.82 \times$ respectively using a batch size of 1024 . When using GTX 1060, we are able to achieve speedups of $123 \times$ in Model A, $61 \times$ in Model B, and 2.8 $\times$ to $4.5 \times$ in CNNbased models (C, D, E). With GTX 1080, which has a larger memory and a faster clock speed, we observe speedups up to $370 \times$ in Model A, $123 \times$ in Model B, and $4.4 \times$ to $7.7 \times$ in CNN-based models (C, D, E).

The results indicate that if the prediction accuracy of Model A is sufficient for a climate simulation, it will provide the greatest calculation speedup using either CPU or GPU. Since NNs with comparable or worse accuracy have been used for simulations ranging from months to years 

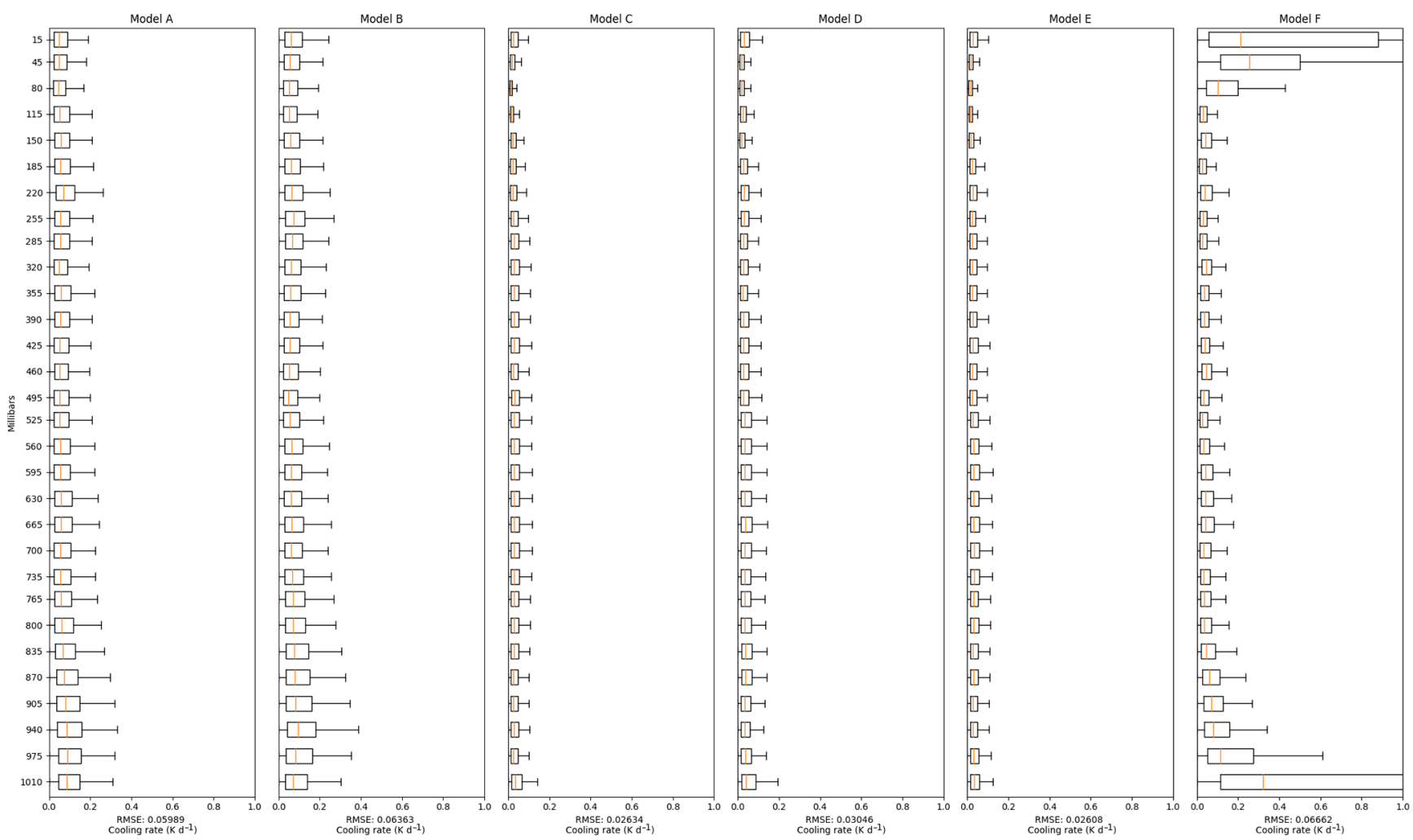

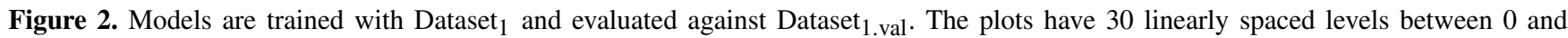
$101300 \mathrm{~Pa}$. The errors from each model are binned to these equally spaced intervals for easier reading. The boxes in the plots present the boxplot of the RT RMSE at every level. Specifically, the boxes describe the 25th (Q1), 50th (Q2), and 75th (Q3) percentile of the RMSEs, while the two whiskers extend from the edges of box to 1.5 times the interquartile range (Q3 - Q1).

(Krasnopolsky et al., 2008; Pal et al., 2019), Model A is a promising candidate for modelling applications since similar performance gains using a full RT code seems to require a complete rewrite for GPUs (Price et al., 2014; Mielikainen et al., 2016; Wang et al., 2020). For simulations requiring a higher accuracy, Model $\mathrm{C}$ provides significant speedups even if a normal GPU is available on the platform.

\subsection{Single-column model simulation}

To explore the ability of the NN model to generalise to new situations, we compare the climate of a single-column model when RRTMG is replaced by the NN Model F (see previous section for a description of Model F). The singlecolumn model uses a diffusive boundary layer (Reed and Jablonowski, 2012), a slab surface of $50 \mathrm{~m}$ thickness which behaves like an oceanic mixed layer, the RRTMG shortwave component, and the Emanuel convection scheme (Emanuel and Zivkovic-Rothman, 1999). The model has no seasonal or diurnal cycle. Carbon dioxide concentration is fixed at $300 \mathrm{ppm}$, and a fixed ozone concentration is prescribed using an observed tropical profile. The model uses pressure as the vertical coordinate and has 60 equally spaced vertical levels between $1013.2 \mathrm{hPa}$ and the model top value at $0 \mathrm{hPa}$. The model time step is $10 \mathrm{~min}$. The tendencies from the various components are stepped forward in time using a third-order explicit Adams-Bashforth scheme.

The model is initialised with a dry, isothermal state. We use RRTMG's longwave component to drive the model until the RMSE between the RRTMG-calculated longwave heating rates and those predicted by Model $\mathrm{F}$ falls below a threshold of $0.5 \mathrm{~K} \mathrm{~d}^{-1}$. Once the errors fall below this value, Model F takes over and RRTMG's longwave component is never used again for the rest of the simulation (shortwave radiation is computed using RRTMG throughout). The switch from RRTMG to Model F happens after around $14 \mathrm{~d}$ of simulation. This simulation is denoted as "RadNet" in Fig. 6. Another simulation continues to use RRTMG longwave radiation until the end of the simulation and is denoted as "RRTMG" in Fig. 6. As discussed subsequently, the RadNet simulation has a bias in the stratosphere, and the temperature profile of the top three levels is constrained to the RRTMG simulation to prevent the simulation from blowing up. Both simulations are run for $2100 \mathrm{~d}$, and equilibrium is reached around $1600 \mathrm{~d}$, with constant temperature and humidity profiles afterwards.

Within the troposphere, both simulations show a realistic moist-adiabatic temperature profile and are in reasonable 

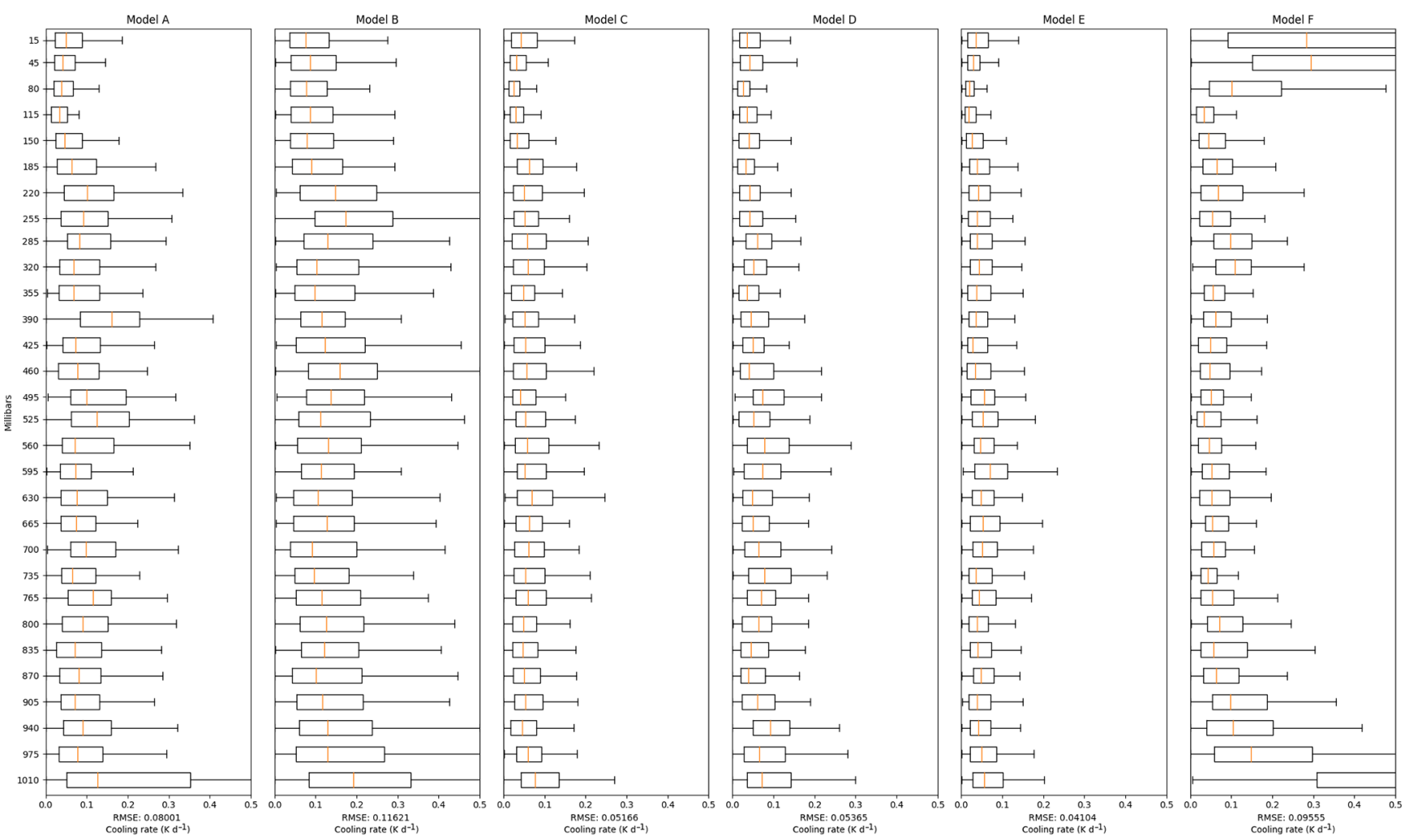

Figure 3. Models are trained with Dataset 2 and evaluated against Dataset $_{1 . v a l}$
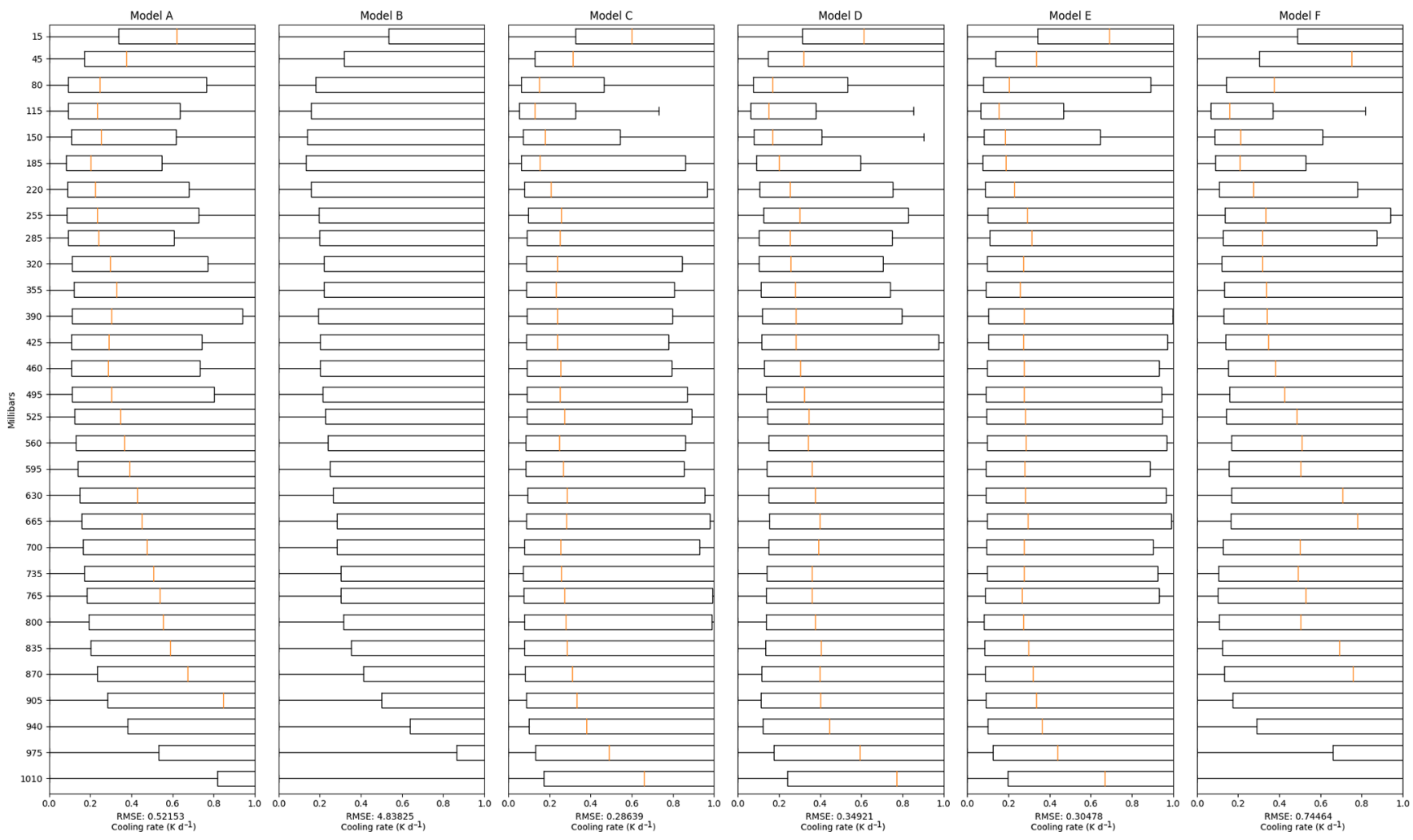

Figure 4. Models are trained with Dataset ${ }_{1}$ and evaluated against Dataset 2 .val 

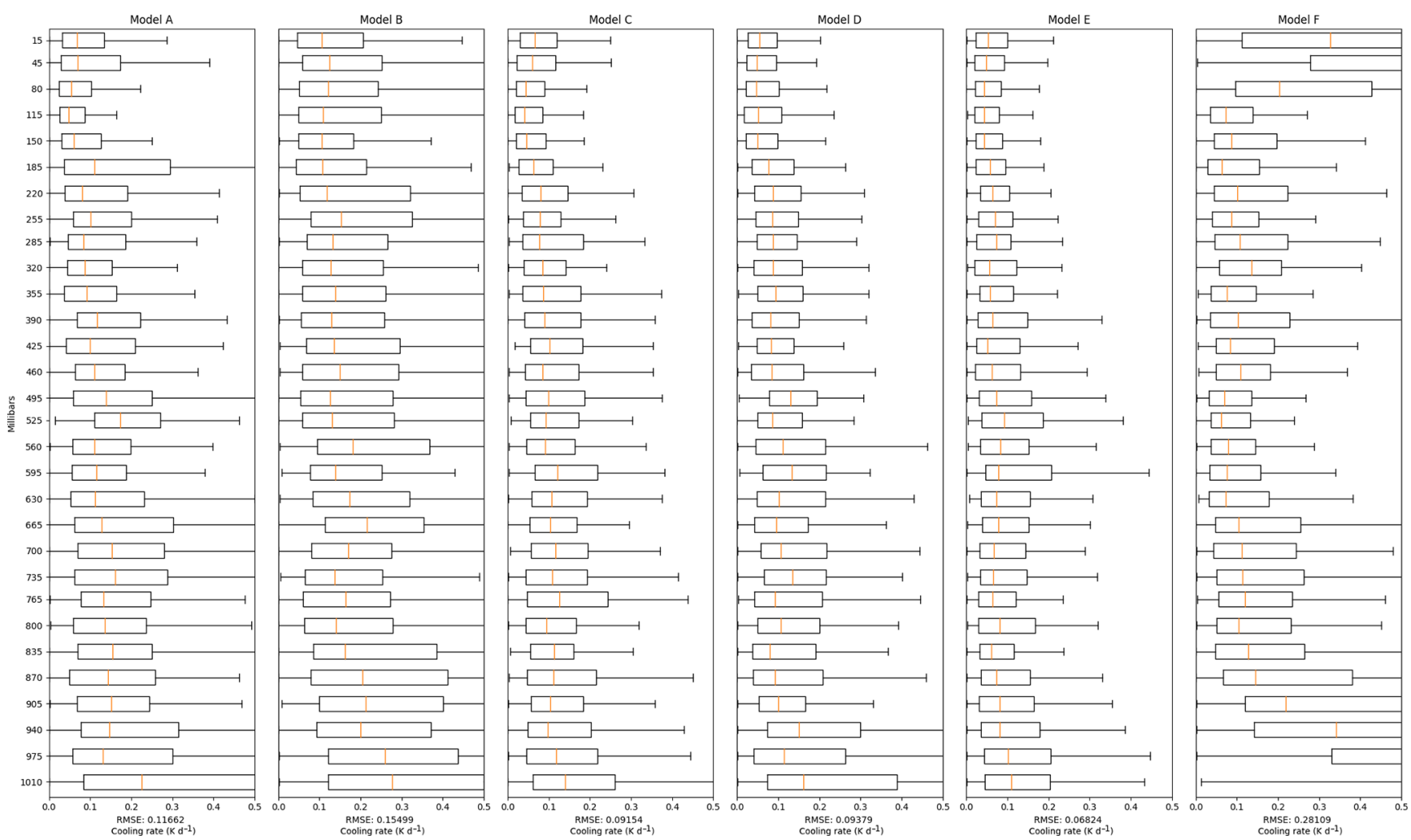

Figure 5. Models are trained with Dataset 2 and evaluated against Dataset $_{2 . v a l}$

Table 2. Speedups when using NN models to predict RTs comparing to calculating RTs using RRTMG. The result for RRTMG is shown for the calculation of a sample in units of milliseconds. Results for NN models are shown as speedups for different batch sizes as compared to the RRTMG calculation on the Xeon CPU.

\begin{tabular}{lrrr|rrr|rrrrr}
\hline Baseline RRTMG & \multicolumn{10}{c}{$0.37 \mathrm{~ms}$} \\
\hline NN model name/hardware & \multicolumn{1}{c}{ Xeon CPU E3-1230 } & \multicolumn{3}{c}{ GTX 1060 } & \multicolumn{4}{c}{ GTX 1080 } \\
\hline NN batch size & 64 & 256 & 1024 & 64 & 256 & 1024 & 64 & 256 & 1024 & 4096 \\
\hline Model A & 5.87 & 10.28 & 10.88 & 18.50 & 61.67 & 123.33 & 16.08 & 61.67 & 123.33 & 370.00 \\
Model B & 1.87 & 2.74 & 2.82 & 13.70 & 37.00 & 61.67 & 14.80 & 46.25 & 74.00 & 123.33 \\
Model C & 0.14 & 0.14 & 0.14 & 3.19 & 4.11 & 4.57 & 4.25 & 5.52 & 7.40 & 7.71 \\
Model D & 0.11 & 0.11 & 0.11 & 2.52 & 3.03 & 3.33 & 3.52 & 4.25 & 5.44 & 5.52 \\
Model E & 0.09 & 0.09 & 0.09 & 2.16 & 2.59 & 2.82 & 2.98 & 3.67 & 4.63 & 4.40 \\
\hline
\end{tabular}

quantitative agreement. However, there are substantial differences in the stratosphere, and the equilibrium position of the tropopause seen in Fig. $6 \mathrm{c}$ in the RadNet simulation is higher by around $50 \mathrm{hPa}$ as compared to the RRTMG simulation. This is because Model F has a cooling bias in the upper atmosphere as seen in Fig. 6d, which makes it convectively unstable, and therefore the tropopause shifts upward. The tropospheric temperature profiles are identical since they are set by the convective parameterisation in such convectively unstable situations.

As the boundary layer fluxes water vapour into the column from the surface, the atmosphere becomes opaque to long- wave radiation in the lower levels, and therefore the longwave cooling is strongest in the level just above the moist, opaque part of the atmosphere. Figure $6 \mathrm{~d}$ shows that the cooling peak predicted by Model $\mathrm{F}$ has a smaller magnitude and is located lower in the atmosphere. The lower cooling rate peak predicted by the NN results in the slower evolution of the RadNet simulation as compared to the RRTMG simulation, resulting in the difference in height between the two simulations (the cooling peak rises over time as the convection tries to eliminate the instability produced by radiative cooling). The cooling peak in the RadNet simulation is situated close to the location of the strongest gradient in water 

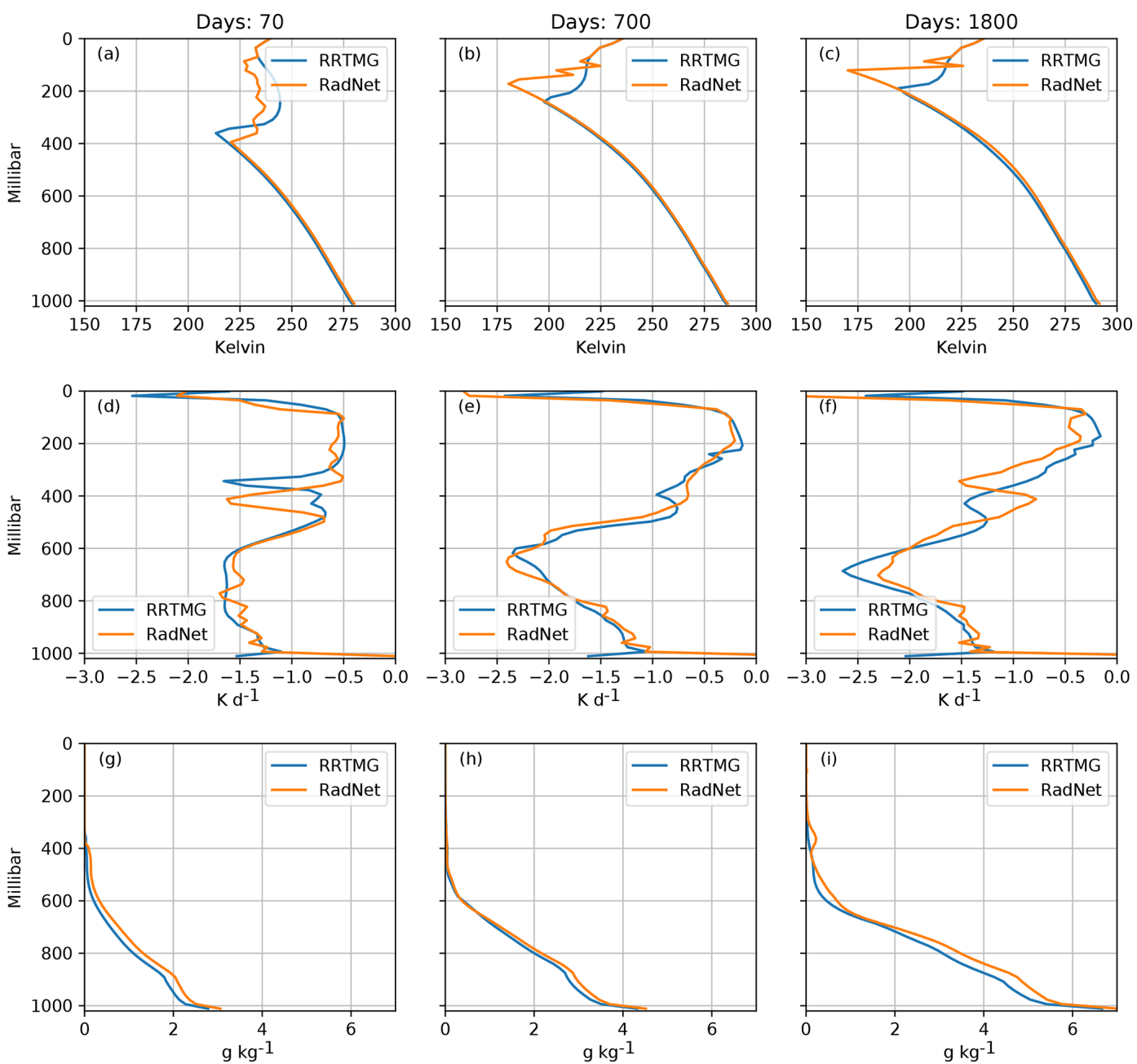

Figure 6. Comparison of the vertical profiles of $(\mathbf{a}-\mathbf{c})$ temperature, $(\mathbf{d}-\mathbf{f})$ longwave heating rates, and (g-i) specific humidity for the RadNet and RRTMG simulations at three different times.

vapour (where the atmosphere transitions from being opaque to transparent to longwave radiation), which is physically accurate. The differences in magnitude are larger slightly earlier in the simulation, where the atmospheric profiles are quite unlike the profiles in the training dataset. It seems unlikely that neural nets can predict such "spiky" profiles correctly since the predicted results tend to be smooth in general. However, the RadNet-predicted profiles provide sufficient cooling to make the atmosphere convectively unstable and eventually mix the entire troposphere of the model.

The $\mathrm{NN}$ has a systematic warm bias in the lowest layer of the model, which may be linked to the interpolation errors discussed previously for Model F. This warm bias results in a slightly warmer surface temperature $(\sim 0.5 \mathrm{~K})$ in the RadNet simulation as seen in Fig. 6c. The warmer profile supports a larger amount of water vapour, and the RadNet simulation has a moist bias in the lower troposphere as well.

We see that small systematic errors in the predicted heating rates can have a nontrivial effect on the simulated climate in a single-column model, especially in the upper layers of the atmosphere. In particular, errors in radiative heating near the tropopause can dramatically change the structure of this part of the atmosphere. The neural network tends to cool the upper atmosphere a little more, making it more convectively unstable and pushing the convection and tropopause higher.

To verify the accuracy of the predicted heating profiles, we use the atmospheric profiles from the RadNet simulation to drive the RRTMG longwave component. The NN and RRTMG heating profiles generated are presented in Fig. 7. The heating profiles predicted by the NN are fairly accurate, especially in the later parts of the simulation when the atmospheric profiles are similar to those in the training sample space. The NN predicts the location of the cooling peak accurately even when the atmospheric profiles are unlike those in the training sample space, though it underestimates the magnitude. RRTMG produces fairly noisy heating profiles in the stratosphere, reflecting the noisy temperature profile simulated by the NN. The noisy stratospheric temperature profile 
appears to be a result of the fact that the training data for Model $\mathrm{F}$ were generated using atmospheric profiles that had additional noise added to them, which results in noisy heating profiles used for training.

\section{Discussion and Conclusions}

Radiative transfer was probably among the first climate model components that neural network models aimed to replace in climate simulations. The evolution of $\mathrm{NN}$ models has paralleled the evolution of $\mathrm{NN}$ architectures themselves, with initial attempts using shallow networks, while recent attempts (including our own) use deep networks. Since both shallow and deep networks seem to perform reasonably well in model simulations (Krasnopolsky et al., 2008; Pal et al., 2019), the question of which type of architecture is more suitable inevitably arises.

In this paper, we have employed two elementary but widely used classes of neural networks, namely feedforward and convolutional neural networks. We believe that the range of model architectures we have presented in this paper is representative within these classes of networks. Our experiments not only explore differences between these two network architectures but also propose a validation workflow:

- traditional validation using metrics such a meansquared error;

- validation against a perturbed dataset, which helps evaluate generalisability of the networks directly;

- validation using a hierarchical climate modelling framework such as climt - simple climate models such as the single-column model help climate scientists not familiar with neural networks evaluate the physical consequences of network architecture choices; the neural network could then be deployed in a more complicated setup such as a general circulation model and evaluated again.

We believe that different methods of validating a neural network are essential to compare different network architectures in a scientifically relevant manner.

Our experiments show the following:

- A larger number of parameters in a neural network lead to slower prediction, as could be expected intuitively.

- More parameters do not always lead to a better prediction accuracy.

Classic convolutional networks provide high accuracy at a higher computational cost. The number of parameters in CNNs can be reduced in multiple ways. For instance, using a one-dimensional convolutional filter could provide performance gains at the expense of losing the correlation information between different input fields like temperature and specific humidity. Similarly, using a larger stride in convolutional layers, using pooling layers in between convolutional layers, and reducing model layers are all ways in which the performance of the classical CNN could be improved. Going beyond the classic architectures we have explored, a variety of architectures have been recently proposed which might increase both accuracy and/or speedup. These architectures include the residual blocks proposed in ResNet (He et al., 2015b) and the depth-wise separable convolution used in MobileNets (Howard et al., 2017) and Xception (Chollet, 2016), among others. Furthermore, EfficientNet (Tan and Le, 2019) has shown that an efficient balancing of network depth, width, and resolution can lead to better performance in terms of prediction accuracy and speed. However, any such reduction of model parameters in CNNs or exploring newer architectures must be accompanied with a rigorous validation procedure, which could be similar to the workflow proposed above.

Recent work in NN theory suggests that the mathematical structure of deep neural networks (a series of linear and nonlinear operators applied sequentially) is especially suited to capture functions which can be expressed as the composition of other functions (Mhaskar and Poggio, 2016; Lin et al., 2017). Radiative transfer conforms to this structure very well; the total radiative heating rate is the sum of heating rates in each spectral band, and the heating rate in each spectral band requires the calculation of absorption coefficients at each model level, each independent of the other. The two-stream approximation and the independent column assumptions introduce additional locality and symmetry requirements, constraining the problem further. This mathematical structure suggests that deep neural networks are a natural choice to approximate RT. Furthermore, the presence of highly localised scattering and absorbing substances such as clouds and water vapour suggests that RT might benefit from a NN structure which is sensitive to localised patterns. This suggests that convolutional NNs might be a better model for RT, and our results confirm this. However, our results also show that using convolutional NNs reduces performance by 50-100 times as compared to feedforward NNs with only a marginal increase in accuracy. Thus, within our evaluation setup, deep feedforward NNs present the best compromise between accuracy and performance. We note that the performance losses we have observed for CNNs could be reduced using a variety of techniques noted in the previous paragraph. The performance-accuracy tradeoff for each of these techniques needs to be evaluated rigorously and provides a promising avenue for future research.

The ability of NNs to generalise to unfamiliar atmospheric profiles seems to be limited as suggested by the cases in which the NNs were validated on the perturbed dataset and the single-column model comparisons. These results bring to question the applicability of NN-based radiative transfer in research configurations where perturbations to the model state or evolution to a wholly new climate state is routinely 

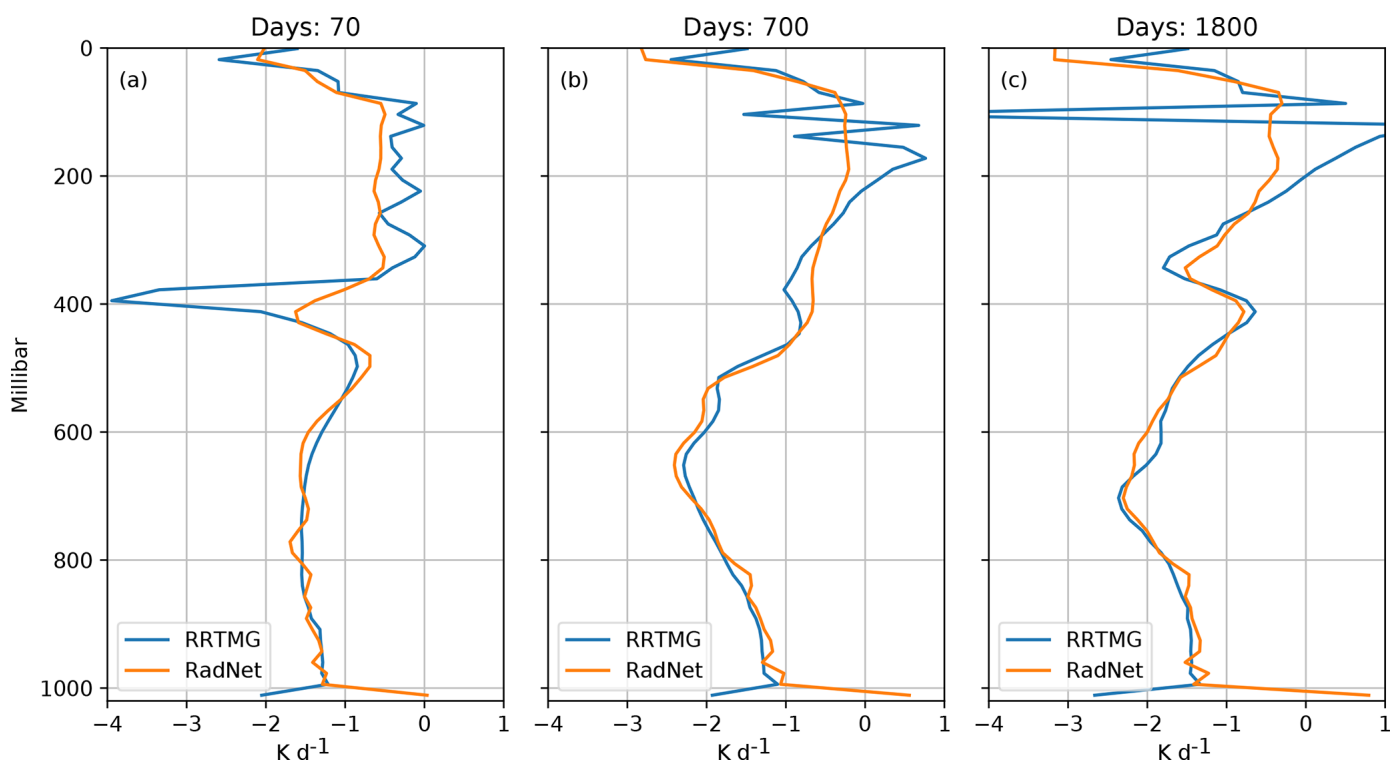

Figure 7. Comparison of the vertical profiles of longwave heating rates predicted by the NN and RRTMG for atmospheric profiles from the RadNet simulation.

performed. Thus, NNs seem to work best in an "operational mode" where the state of the climate or weather prediction model is not expected to change dramatically as compared to the training set. The approach of adding of noise to improve NNs' ability to generalise beyond the training sample has a long history (Sietsma and Dow, 1991). However, our results show that adding noise to the training dataset results in noisy temperature profiles in simulations, especially in the stratosphere where the temperature profile is closer to pure radiative equilibrium.

The dramatic performance gains when using commodity GPUs makes the use of NNs all the more attractive given that most future high-performance computing configurations will include both GPUs and CPUs. NNs allow batching of multiple atmospheric profiles during matrix multiplications, which allows large performance gains. Such batching is not feasible for an actual RT calculation, and each atmospheric profile has to be handled individually. This may be the reason why rewrites of RRTMG for GPUs (Price et al., 2014; Mielikainen et al., 2016; Wang et al., 2020) give similar performance gains to what we have achieved in our setup using NNs. We note that the comparison between RadNet and rewrites of RRTMG for GPUs does not take into consideration differences in GPU architectures and batch sizes, which could change the exact numbers obtained. However, our results highlight the difference that GPUs make in accelerating RadNet.

Another method to assess the ability of NNs to generalise is to actually build a climate model which includes the NN as a component. Since single-column models have no diffusion built in and cannot transport energy horizontally, we believe that they constitute a tougher test case for NNs as compared to GCMs. The lack of dynamics also makes the results easier to interpret. In our test case, we see that the errors in prediction by the $\mathrm{NN}$ have a larger impact in the stratosphere than the troposphere due to the tight control of the tropospheric lapse rate by moist convection. The initial atmospheric profile - dry and isothermal - is quite different from the profiles in the training sample space. While the errors in the initial part of the simulation are larger, the NN predicts physically realistic heating profiles with slight differences in location and magnitude. Such physically plausible behaviour in situations quite different from those the NN was trained on gives us confidence that NNs can indeed be used as climate model components in the future. However, it is clear that better strategies for data preparation, selection of $\mathrm{NN}$ architecture, and testing trained NNs are required to improve NN performance and enable scientists to interpret their impact on climate model simulations.

Code availability. The code used for training RadNet and the Jupyter notebook which simulates the single-column model is available at https://doi.org/10.5281/zenodo.3884964 (Liu et al., 2019).

The ERA-Interim data can be downloaded from https: //apps.ecmwf.int/datasets/data/interim-full-daily/levtype=sfc/ (last access: December 2019, Dee et al., 2011).

Author contributions. YL designed, trained, and validated the neural networks, prepared the input data, and participated in design of the project and writing the paper. RC participated in design of the project and writing the paper. JMM wrote the climt code to generate the data, interfaced RadNet to the single-column model, analysed the simulations, and participated in writing the paper. 
Competing interests. The authors declare that they have no conflict of interest.

Acknowledgements. All authors were funded by the Swedish eScience Research Centre (SeRC).

Financial support. This research has been supported by the Swedish e-Science Research Centre (SeRC).

The article processing charges for this open-access publication were covered by Stockholm University.

Review statement. This paper was edited by Juan Antonio Añel and reviewed by Tomás Fernández-Pena and one anonymous referee.

\section{References}

Brenowitz, N. D. and Bretherton, C. S.: Prognostic Validation of a Neural Network Unified Physics Parameterization, Geophys. Res. Lett., 45, 6289-6298, https://doi.org/10.1029/2018GL078510, 2018.

Brenowitz, N. D. and Bretherton, C. S.: Spatially Extended Tests of a Neural Network Parametrization Trained by Coarse-Graining, J. Adv. Model. Earth Sy., 11, 2728-2744, https://doi.org/10.1029/2019MS001711, 2019.

Chevallier, F., Chéruy, F., Scott, N. A., and Chédin, A.: A Neural Network Approach for a Fast and Accurate Computation of a Longwave Radiative Budget, J. Appl. Meteorol., 37, 1385-1397, https://doi.org/10.1175/15200450(1998)037<1385:ANNAFA>2.0.CO;2, 1998.

Chollet, F.: Xception: Deep Learning with Depthwise Separable Convolutions, CoRR, abs/1610.02357, 2016.

Dee, D. P., Uppala, S. M., Simmons, A. J., Berrisford, P., Poli, P., Kobayashi, S., Andrae, U., Balmaseda, M. A., Balsamo, G., Bauer, P., Bechtold, P., Beljaars, A. C. M., van de Berg, I., Biblot, J., Bormann, N., Delsol, C., Dragani, R., Fuentes, M., Greer, A. J., Haimberger, L., Healy, S. B., Hersbach, H., Holm, E. V., Isaksen, L., Kallberg, P., Kohler, M., Matricardi, M., McNally, A. P., Mong-Sanz, B. M., Morcette, J.-J., Park, B.-K., Peubey, C., de Rosnay, P., Tavolato, C., Thepaut, J. N., and Vitart, F.: The ERAInterim reanalysis: Configuration and performance of the data assimilation system, Q. J. Roy. Meteorol. Soc., 137, 553-597, https://doi.org/10.1002/qj.828, 2011.

Dennis, J. M. and Loft, R. D.: Refactoring Scientific Applications for Massive Parallelism, in: Numerical Techniques for Global Atmospheric Models, Lecture Notes in Computational Science and Engineering, Springer, Berlin, Heidelberg, 539-556, https://doi.org/10.1007/978-3-642-11640-7_16, 2011.

Dueben, P. D. and Bauer, P.: Challenges and design choices for global weather and climate models based on machine learning, Geosci. Model Dev., 11, 3999-4009, https://doi.org/10.5194/gmd-11-3999-2018, 2018.

Emanuel, K. A. and Zivkovic-Rothman, M.: Development and Evaluation of a Convection Scheme for Use in Climate Mod- els, J. Atmos. Sci., 56, 1766-1782, https://doi.org/10.1175/15200469(1999)056<1766:DAEOAC>2.0.CO;2, 00553, 1999.

$\mathrm{Fu}$, Q. and Liou, K. N.: On the Correlated k-Distribution Method for Radiative Transfer in Nonhomogeneous Atmospheres, J. Atmos. Sci., 49, 2139-2156, https://doi.org/10.1175/15200469(1992)049<2139:OTCDMF>2.0.CO;2, 1992.

Gardner, M. and Dorling, S.: Artificial Neural Networks (The Multilayer Perceptron) - A Review of Applications in the Atmospheric Sciences, Atmos. Environ., 32, 2627-2636, 1998.

Gentine, P., Pritchard, M., Rasp, S., Reinaudi, G., and Yacalis, G.: Could Machine Learning Break the Convection Parameterization Deadlock?, Geophys. Res. Lett., 45, 5742-5751, https://doi.org/10.1029/2018GL078202, 2018.

Goodfellow, I., Bengio, Y., and Courville, A.: Deep learning, MIT press, 2016.

He, K., Zhang, X., Ren, S., and Sun, J.: Delving Deep into Rectifiers: Surpassing Human-Level Performance on ImageNet Classification, CoRR, abs/1502.01852, $2015 \mathrm{a}$.

He, K., Zhang, X., Ren, S., and Sun, J.: Deep Residual Learning for Image Recognition, CoRR, abs/1512.03385, 2015b.

Howard, A. G., Zhu, M., Chen, B., Kalenichenko, D., Wang, W., Weyand, T., Andreetto, M., and Adam, H.: MobileNets: Efficient Convolutional Neural Networks for Mobile Vision Applications, CoRR, abs/1704.04861, 2017.

Iacono, M. J., Delamere, J. S., Mlawer, E. J., Shephard, M. W., Clough, S. A., and Collins, W. D.: Radiative forcing by longlived greenhouse gases: Calculations with the AER radiative transfer models, J. Geophys. Res.-Atmos., 113, D13103, https://doi.org/10.1029/2008JD009944, 2008a.

Iacono, M. J., Delamere, J. S., Mlawer, E. J., Shephard, M. W., Clough, S. A., and Collins, W. D.: Radiative forcing by longlived greenhouse gases: Calculations with the AER radiative transfer models, J. Geophys. Res.-Atmos., 113, D13103, https://doi.org/10.1029/2008JD009944, 2008b.

Innamorati, C., Ritschel, T., Weyrich, T., and Mitra, N. J.: Learning on the Edge: Explicit Boundary Handling in CNNs, CoRR, abs/1805.03106, 2018.

Kingma, D. P. and Ba, J.: Adam: A Method for Stochastic Optimization, CoRR, abs/1412.6980, 2014.

Krasnopolsky, V. M., Fox-Rabinovitz, M. S., and Chalikov, D. V.: New Approach to Calculation of Atmospheric Model Physics: Accurate and Fast Neural Network Emulation of Longwave Radiation in a Climate Model, Mon. Weather Rev., 133, 1370-1383, https://doi.org/10.1175/MWR2923.1, 2005.

Krasnopolsky, V. M., Fox-Rabinovitz, M. S., and Belochitski, A. A.: Decadal Climate Simulations Using Accurate and Fast Neural Network Emulation of Full, Longwave and Shortwave, Radiation, Mon. Weather Rev., 136, 3683-3695, https://doi.org/10.1175/2008MWR2385.1, 2008.

Krasnopolsky, V. M., Fox-Rabinovitz, M. S., Hou, Y. T., Lord, S. J., and Belochitski, A. A.: Accurate and Fast Neural Network Emulations of Model Radiation for the NCEP Coupled Climate Forecast System: Climate Simulations and Seasonal Predictions, Mon. Weather Rev., 138, 1822-1842, https://doi.org/10.1175/2009MWR3149.1, 2009.

Krizhevsky, A., Sutskever, I., and Hinton, G. E.: ImageNet Classification withDeep Convolutional Neural Networks, in: Proceedings of the 25th International Conference on Neural Information Processing Systems - Volume 1, NIPS'12, 1097- 
1105, Curran Associates Inc., USA, available at: http://dl.acm. org/citation.cfm?id=2999134.2999257 (last access: 1 December 2019), 2012a.

Krizhevsky, A., Sutskever, I., and Hinton, G. E.: Imagenet classification with deep convolutional neural networks, in: Advances in neural information processing systems, 1097-1105, 2012b.

Krizhevsky, A., Sutskever, I., and Hinton, G. E.: ImageNet Classification with Deep Convolutional Neural Networks, Commun. ACM, 60, 84-90, 2017.

Lin, H. W., Tegmark, M., and Rolnick, D.: Why Does Deep and Cheap Learning Work So Well?, J. Stat. Phys., 168, 1223-1247, https://doi.org/10.1007/s10955-017-1836-5, 2017.

Liu, Y., Monteiro, J. M., and Caballero, R.: RadNet release for GMD, Zenodo, https://doi.org/10.5281/zenodo.3884964, 2019.

Malik, M., Grosheintz, L., Mendonça, J. M., Grimm, S. L., Lavie, B., Kitzmann, D., Tsai, S.-M., Burrows, A., Kreidberg, L., Bedell, M., Bean, J. L., Stevenson, K. B., and Heng, K.: Helios: An Open-Source, Gpu-Accelerated Radiative Transfer Code For Self-Consistent Exoplanetary Atmospheres, Astron. J., 153, 56, https://doi.org/10.3847/1538-3881/153/2/56, 2017.

Meador, W. E. and Weaver, W. R.: Two-Stream Approximations to Radiative Transfer in Planetary Atmospheres: A Unified Description of Existing Methods and a New Improvement, J. Atmos. Sci., 37, 630-643, https://doi.org/10.1175/15200469(1980)037<0630:TSATRT>2.0.CO;2, 1980.

Mhaskar, H. N. and Poggio, T.: Deep vs. shallow networks: An approximation theory perspective, Anal. Appl., 14, 829-848, https://doi.org/10.1142/S0219530516400042, 2016.

Mielikainen, J., Price, E., Huang, B., Huang, H. A., and Lee, T.: GPU Compute Unified Device Architecture (CUDA)based Parallelization of the RRTMG Shortwave Rapid Radiative Transfer Model, IEEE J. Sel. Top. Appl., 9, 921-931, https://doi.org/10.1109/JSTARS.2015.2427652, 2016.

Monteiro, J. M. and Caballero, R.: The Climate Modelling Toolkit, in: Proceedings of the 15th Python in Science Conference, Austin, USA, 69-74, available at: http://conference.scipy.org/ proceedings/scipy2016/joy_monteiro.html (last access: 1 December 2019), 2016.

Monteiro, J. M., McGibbon, J., and Caballero, R.: sympl (v. 0.4.0) and climt (v. 0.15.3) - towards a flexible framework for building model hierarchies in Python, Geosci. Model Dev., 11, 37813794, https://doi.org/10.5194/gmd-11-3781-2018, 2018.

O'Gorman, P. A. and Dwyer, J. G.: Using Machine Learning to Parameterize Moist Convection: Potential for Modeling of Climate, Climate Change, and Extreme Events, J. Adv. Model. Earth Sy., 10, 2548-2563, https://doi.org/10.1029/2018MS001351, 2018.

O'Shea, K. and Nash, R.: An Introduction to Convolutional Neural Networks, CoRR, abs/1511.08458, 2015.

Pal, A., Mahajan, S., and Norman, M. R.: Using Deep Neural Networks as Cost-Effective Surrogate Models for SuperParameterized E3SM Radiative Transfer, Geophys. Res. Lett., 46, 6069-6079, https://doi.org/10.1029/2018GL081646, 2019.

Palmer, T. N.: More reliable forecasts with less precise computations: a fast-track route to cloud-resolved weather and climate simulators?, Philos. T. R. Soc. A, 372, 20130391, https://doi.org/10.1098/rsta.2013.0391, 2014.
Pincus, R. and Stevens, B.: Paths to accuracy for radiation parameterizations in atmospheric models, J. Adv. Model. Earth. Sy., 5, 225-233, https://doi.org/10.1002/jame.20027, 2013.

Price, E., Mielikainen, J., Huang, M., Huang, B., Huang, H. A., and Lee, T.: GPU-Accelerated Longwave Radiation Scheme of the Rapid Radiative Transfer Model for General Circulation Models (RRTMG), IEEE J. Sel. Top. Appl., 7, 3660-3667, https://doi.org/10.1109/JSTARS.2014.2315771, 2014.

Rasp, S., Pritchard, M. S., and Gentine, P.: Deep learning to represent subgrid processes in climate models, P. Natl. Acad. Sci. USA, 115, 9684-9689, https://doi.org/10.1073/pnas.1810286115, 2018.

Reed, K. A. and Jablonowski, C.: Idealized tropical cyclone simulations of intermediate complexity: a test case for AGCMs, J. Adv. Model. Earth Sy., 4, M04001, https://doi.org/10.1029/2011MS000099, 2012.

San, O. and Maulik, R.: Extreme learning machine for reduced order modeling of turbulent geophysical flows, Phys. Rev. E, 97, 042322, https://doi.org/10.1103/PhysRevE.97.042322, 2018.

Scher, S.: Toward Data-Driven Weather and Climate Forecasting: Approximating a Simple General Circulation Model With Deep Learning, Geophys. Res. Lett., 45, 12616-12622, https://doi.org/10.1029/2018GL080704, 2018.

Schneider, T., Lan, S., Stuart, A., and Teixeira, J.: Earth system modeling 2.0: A blueprint for models that learn from observations and targeted high-resolution simulations, Geophys. Res. Lett., 44, 12-396, 2017.

Sietsma, J. and Dow, R. J. F.: Creating artificial neural networks that generalize, Neural Networks, 4, 67-79, https://doi.org/10.1016/0893-6080(91)90033-2, 1991.

Simonyan, K. and Zisserman, A.: Very deep convolutional networks for large-scale image recognition, arXiv preprint arXiv:1409.1556, 2014.

Tan, M. and Le, Q. V.: EfficientNet: Rethinking Model Scaling for Convolutional Neural Networks, CoRR, abs/1905.11946, 2019.

Wang, Y., Zhao, Y., Jiang, J., and Zhang, H.: A Novel GPU-Based Acceleration Algorithm for a Longwave Radiative Transfer Model, Appl. Sci., 10, 649, https://doi.org/10.3390/app10020649, 2020.

Wu, Y., Schuster, M., Chen, Z., Le, Q. V., Norouzi, M., Macherey, W., Krikun, M., Cao, Y., Gao, Q., Macherey, K., Klingner, J., Shah, A., Johnson, M., Liu, X., Kaiser, L., Gouws, S., Kato, Y., Kudo, T., Kazawa, H., Stevens, K., Kurian, G., Patil, N., Wang, W., Young, C., Smith, J., Riesa, J., Rudnick, A., Vinyals, O., Corrado, G., Hughes, M., and Dean, J.: Google's Neural Machine Translation System: Bridging the Gap between Human and Machine Translation, CoRR, abs/1609.08144, 2016.

Yuval, J. and O'Gorman, P. A.: Use of machine learning to improve simulations of climate, arXiv:2001.03151, 2020. 